on Levodopa (Group B) showed significant decrease (inhibition) in brainstem reflexes amplitude after Levodopa.

Conclusion Different patterns of cortical and brainstem activity, reflecting different mechanisms of compensation with Levodopa intake, can differentiate dysphagic PD groups. Moreover, physiology was negatively affected by L-dopa in groups with inhibition of brainstem reflexes. Our novel brain stimulation data demonstrate the dysphagia in PD patients is associated with altered cortical and brainstem activity, modulated by L-dopa differentially across the PD groups, providing the platform for research on rehabilitation for dysphagia in PD.

Disclosure of Interest None Declared

\section{OC-035 MECHANISM OF LACTOSE INTOLERANCE IN IRRITABLE BOWEL SYNDROME: ROLE OF ANXIETY, MUCOSAL IMMUNITY AND VISCERAL HYPERSENSITIVITY}

doi:10.1136/gutjnl-2013-304907.035

1,"Y Long, ${ }^{1} \mathrm{~J}$ Yang, ${ }^{1} \mathrm{Y}$ Zhu, ${ }^{1} \mathrm{Y}$ Cong, ${ }^{1} \mathrm{H}$ Chu, ${ }^{1} \mathrm{~N}$ Dai, ${ }^{2} \mathrm{M}$ Fried, ${ }^{3} \mathrm{M}$ Fox. ${ }^{1}$ Department of Gastroenterology, Sir Run Run Shaw Hospital, School of Medicine, Zhejiang University, Hangzhou, China; 'Division of Gastroenterology and Hepatology, Zurich University Hospital, Zurich, Switzerland; ${ }^{3}$ NIHR Nottingham Digestive Diseases Biomedical Research Unit, Nottingham University Hospitals, Nottingham, UK

Introduction Many patients, especially those with Irritable Bowel Syndrome (IBS), report food intolerance; however the mechanism underlying these "functional digestive symptoms" is unknown. We assessed the role of psychological factors, mucosal immune activation and visceral sensitivity on the development of lactose intolerance (LI) after ingestion of $20 \mathrm{~g}$ lactose in IBS patients and healthy controls (HCs) with lactase deficiency: a validated, clinical experimental model of intolerance to poorly absorbed, fermentable foods (Yang et al Clin Gastro Hep 2013 in publication)

Methods IBS-D patients meeting Rome III criteria $(n=277)$ and age/sex matched HCs $(n=64)$ underwent a $20 \mathrm{~g}$ lactose hydrogen breath test (LHBT) with measurement of hydrogen production, abdominal distention, and LI symptoms. Hospital Anxiety and Depression Score assessed psychologic state. Barostat measured rectal sensitivity. Additionally, 55 IBS-D patients and $18 \mathrm{HCs}$ completed colonoscopy with colon and terminal ileum (TI) biopsies for quantification of mast cells (MCs), T lymphocytes, and enterochromaffin cells (ECC).

Results Hydrogen production and distention were similar in IBS patients and HCs during LHBT; however LI symptoms were more frequent in IBS ( $54 \%$ vs. $28 \%, P<0.001)$, including bloating $(39 \%$ vs. $14 \%, P<0.001)$, borborygmi (39\% vs. $22 \%, P=0.010)$, pain $(31 \%$ vs. $11 \%, P=0.001)$ and diarrhoea ( $29 \%$ vs. $9 \%, P=0.001)$. IBS patients were more anxious $(\mathrm{p}<0.001)$ and had higher rectal sensitivity than HCs $(P=0.001)$. Multivariate analysis indicated that hydrogen production increased the likelihood of bloating (OR2.2 (95\%CI 1.1-4.4), $P=0.028)$ and borborygmi (OR12.4 (3.3-45.8), $P<0.001)$, but not objective distention $(P=0.673)$. Visceral hypersensitivity also associated with bloating (OR6.6 (1.7-25.0), $P=0.005)$ and total symptom score (OR3.7 (1.3-10.9, $P=0.014)$. A planned subanalysis showed that, compared to IBS patients with no symptoms, those with LI were more anxious $(p=0.045)$ and more likely to have visceral hypersensitivity $(p<0.001)$.

In those that had colonoscopy, IBS patients with LI $(25 / 55$ $(45 \%)$ ) had increased MCs ( $p<0.006)$, T-cells and ECC (both $\mathrm{p}<0.05$ ) in proximal colonic and TI mucosa compared to patients without LI and HCs. Multivariate analysis indicated that total LI symptom score was associated with anxiety $(r=0.519, P<0.001)$, MCs in terminal ileum $(\mathrm{r}=0.650, P<0.001)$ and visceral sensitivity $(\mathrm{r}=0.629, P<0.001)$.

Conclusion Gas production and sensitivity to luminal distension both contribute to digestive symptoms after lactose ingestion in patients with lactase deficiency. IBS-D patients with LI are characterised by anxiety, evidence of mucosal immune activation and visceral hypersensitivity.

Disclosure of Interest None Declared

\section{OC-036 NEURONAVIGATED REPETITIVE CEREBELLAR STIMULATION PRODUCES LONG-LASTING ACTIVATION OF HUMAN CORTICAL SWALLOWING PROJECTIONS}

doi:10.1136/gutjnl-2013-304907.036

1,"D H Vasant, 'S Mistry, 'V Jayasekeran, 'E Michou, 'S Hamdy. 'Gastrointestinal Centre, Institute of Inflammation and Repair, Manchester Academic Health Sciences Centre, University of Manchester, Salford Royal NHS Foundation Trust, Salford, UK

Introduction Animal studies, human brain imaging and more recently Transcranial Magnetic Stimulation (TMS) suggest a role for the cerebellum in human swallowing. Moreover, paired-pulse cerebellar-cortical TMS delivered in rapid succession $(50-200 \mathrm{~ms}$ intervals) facilitates pharyngeal motor cortex excitability. We therefore hypothesised that high-frequency $(5-20 \mathrm{~Hz})$ repetitive TMS (rTMS) of the cerebellum could modulate pharyngeal motor cortex excitability producing long-lasting changes that may prove to be therapeutically useful for dysphagia after stroke.

Methods In 17 healthy adults (6 female, age range 18-61 yrs), anatomical MR brain scans were acquired. Thereafter participants were intubated with an intraluminal catheter to record pharyngeal electromyography and underwent TMS cortical mapping with neuronavigation to co-localise pharyngeal motor representation bilaterally, hand motor cortex and the cerebellar site which evoked the largest pharyngeal motor response. Subjects were then randomised to receive one of 5 neuronavigated cerebellar rTMS interventions (Sham, $1 \mathrm{~Hz}, 5 \mathrm{~Hz}, 10 \mathrm{~Hz}$ and $20 \mathrm{~Hz}$, at least 1 week apart) to the cerebellar site evoking the largest baseline pharyngeal responses to single-pulse cerebellar TMS. Bihemispheric pharyngeal cortical excitability (ipsilateral and contralateral cortex to cerebellum site) was measured at baseline and for up to one hour post cerebellar rTMS intervention. Abductor pollicis brevis (APB) recordings were used as control. Interventional data were compared to sham using repeated measures ANOVA.

Results Cerebellar rTMS was tolerated well and delivered at an average intensity of $55 \%$ of stimulator output. Compared to Sham, $10 \mathrm{~Hz}$ cerebellar rTMS increased pharyngeal cortical excitability ( $\mathrm{F}$ $\left.(1.16)=8.3,{ }^{*} p=0.01\right)$, with maximal size and durational effects seen primarily in the contralateral pharyngeal cortex $(+72 \%$, ${ }^{* *} p=0.02$, Figure 1). By contrast, $1 \mathrm{~Hz}(\mathrm{~F}(1.16)=0.3, p=0.60), 5 \mathrm{~Hz}$ $(\mathrm{F}(1.16)=0.5, p=0.48)$, and $20 \mathrm{~Hz} \operatorname{rTMS}(\mathrm{F}(1.16)=1.3, p=0.27)$ cerebellar conditioning did not significantly alter pharyngeal excitability compared to Sham. APB responses were not significantly different to sham after any intervention.

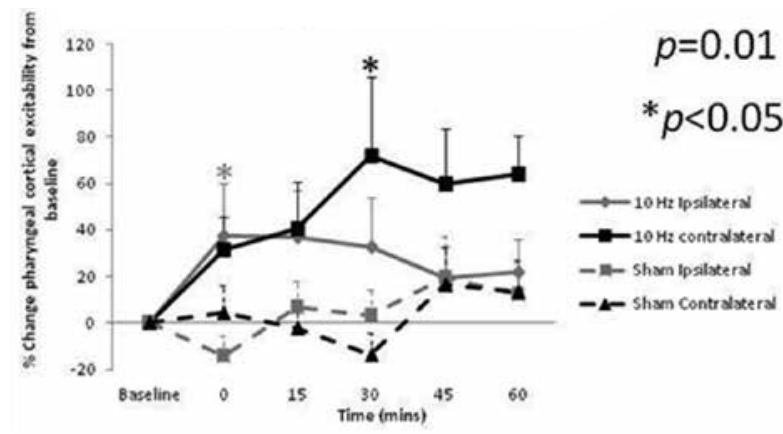

Abstract 0C-036 Figure 1 Effects of $10 \mathrm{~Hz}$ Cerebellar rTMS on pharyngeal cortical excitability. 